\title{
Face Recognition using Genetic Algorithm and Neural Networks
}

\author{
Mahendra Pratap Panigrahy \\ Associate Professor, ECE \\ Institute of Technology Roorkee \\ Haridwar, Uttarkhand, India
}

\author{
Neeraj Kumar \\ Assistant Professor, CSE \\ Institute of Technology Roorkee \\ Haridwar, Uttarkhand, India
}

\begin{abstract}
This article deals with the combinations basics of Genetic Algorithm (GA) and Back Propagation Neural Networks (BPNN) and their applications in Pattern Recognition or for Face Recognition problems. Images have a huge information and characteristics quantities. Until today, a complete efficient mechanism to extract these characteristics in an automatic way is yet not possible. Referring to facial images, its detection in an image is a problem that requires a meticulous investigation due to its high complexity. Here we should investigate the aspects of genetic in face recognition. Genetic Algorithms (GA's) are characterized as one search technique inspired by Darwin Evolutionist Theory. Genetic Algorithm is efficient in reducing computation time for a huge heapspace. Face recognition from a very huge Heap-space is a time consuming task hence genetic algorithm based approach is used to recognize the unidentified -image within a short span of time. BPNN can be viewed as computing models inspired by the structure and function of the biological neural network. See that the training process does not have a single call to a training function, but the network was trained several times on various input ideal and noisy images, the images which contents face. In this case training a network on different sets of noisy images forced the network to learn how to deal with noise, a common problem in the real world. These models are expected to deal with problem solving in a manner different from conventional computing. A distinction is made between pattern and data to emphasize the need for developing pattern processing systems to address pattern recognition tasks.
\end{abstract}

\section{General Terms}

Facial Images, Darwin Evolutionist Theory.

\section{Keywords}

Genetic Algorithm (GA); Back Propagation Neural Networks (BPNN); Face Recognition; Pattern Recognition; Biological Neural Network.

\section{INTRODUCTION}

The Digital Images are having such huge information and characteristics as these are more complex, so until today, a complete efficient mechanism to extract these characteristics in an automatic way is not yet possible. Referring to facial images, its detection in an image is a problem that requires lots of investigation due to its high complexivity and irregularities. The usage of these images is very broad and wide, such as the security systems case, where it can be used for remote conference, search in database, people identification etc. In today's scenario the increase of terrorist attacks, motivates countless works in this area. Security cameras are placed in supermarkets, shopping centers, subways, downtown, parks, buses, and so on, trying to help for the identification of guilty people, besides reducing crimes and illegal things that happening in the surrounding. Unfortunately, until now, security systems need human supervision, which causes significant failures. Here, we propose an investigation of the Genetic Algorithms technique application in facial detection, which will solve the one step for face recognition. According to the Genetic Algorithms (GA's) are characterized as one search technique inspired by Darwin Evolutionary Theory, shaped using some selection mechanisms used in Nature, according with that individuals who are abler in a population are those who have more survival possibility, when adapting themselves more easily to the changes that occur in their habitats. Human problem solving is basically a pattern processing problem but merely a data processing problem. In any pattern recognition task humans perceive patterns in the input data and manipulate the pattern directly. Here we discuss attempts at developing computing models based on artificial neural networks (ANN) to deal with various pattern recognition situations in real life with the help of Genetic Algorithm.

\section{FACE DETECTION}

An important task to be carried out in the Systems of Recognition of Faces is to detect the presence of a face in a determined region of the image. To detect the face before trying to recognize it saves a lot of work, as only a restricted region of the image is analyzed, opposite to many algorithms which work considering the whole image. The design and implementation of the Face Recognition System (FRS) is subdivided into two main parts. The first part is Image Processing and the second part is Recognition Techniques. The image processing part consists of Face image acquisition through scanning, Image enhancement, Image clipping, Filtering, Edge detection and Feature extraction. The second part consists of the Artificial Intelligence which is the Artificial Neural Network will solve the purpose of recognition part.

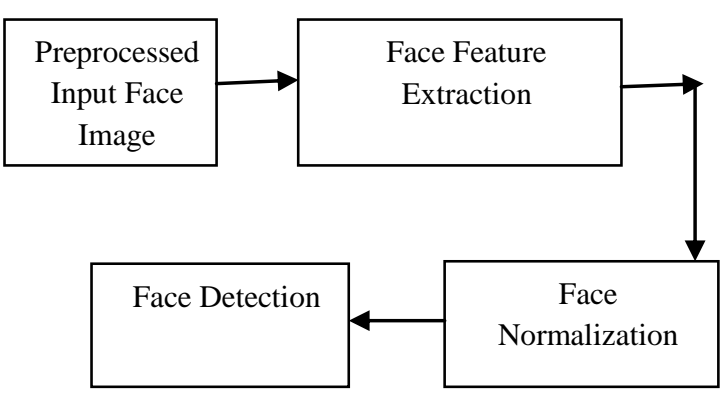

Figure-1: Basic Block Diagram of Face Detection System 


\subsection{Genetic Algorithms}

The Genetic Algorithms (GA's) are characterized by a search technique inspired in the Evolutionist Theory by Darwin, uses some selection mechanism. Where individuals that is the chromosomes more adapted of a population are the ones that have more survival chances, by getting used easily to changes that occur in its environment. This makes the algorithm strong and fast, being designate to a determined type of optimization, where the search space is too big and the conventional methods become inefficient. Another GA's important characteristic is that they result a set of solutions and not only one solution.

An algorithm and the cycle of the GA are described in the following steps:

a. Start a population of $\mathrm{N}$ size, with chromosomes generated randomly.

b. Apply fitness to each chromosome of population.

c. Make new chromosomes through crossings of selected chromosomes of this population. Apply recombination and mutation in these chromosomes.

d. Eliminate members of old population, in order to have space to insert these new chromosomes, keeping the population with the same $\mathrm{N}$ chromosomes.

e. Apply fitness in these chromosomes and insert them in the population.

f. If the ideal solution will be found or, if the time (or generation number) depleted, return the chromosome with best fitness. Otherwise, come back to the step c.

The above algorithm can be represented as in the Flow Charts as in Figure-2.



Figure-2: Flow Chart of Proposed GA

\subsection{Artificial Neural Network}

Artificial Neural Networks (ANN) is very well known, powerful and robust classification techniques that are used for real- valued, discrete-valued and vector valued functions. Here we will apply an ANN which uses the Two Layer Back Propagation Algorithm for learning. The back propagation algorithm is used to minimize the error functions.
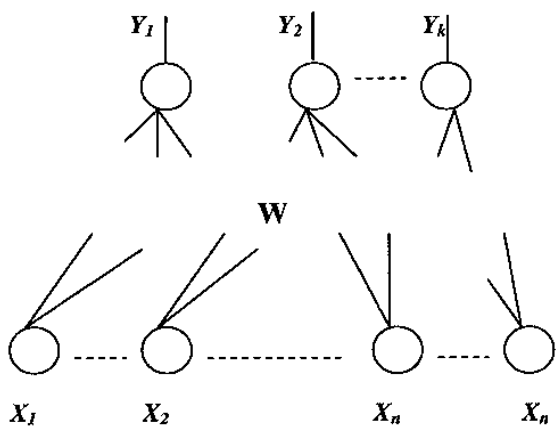

Figure 3: Two Stages ANN

Here the example is a Face Recognition, $<\left[\mathrm{X}_{1}, \ldots \ldots \ldots, \mathrm{X}_{\mathrm{n}-1}\right.$, $\mathrm{Xn+}] \mathrm{T}, \mathrm{Y}_{\mathrm{K}}>$. Where $\left[\mathrm{X}_{1}, \ldots \ldots . ., \mathrm{Xn}-1, \mathrm{Xn+1}\right] \mathrm{T}$ is the input (feature vector the face) to the ANN and YK is the output (of the face). The output is a Boolean Value but not a Probability. The Probability is fit to a Boolean Value by using a Sigmoid Function after the ANN output. The approximated probabilistic output, $\mathrm{o}^{\prime}=\mathrm{f}(\mathrm{o}(\mathrm{I}))$, where $\mathrm{I}$ is an input session, $\mathrm{o}(\mathrm{I})$ corresponds to $\mathrm{Y}_{\mathrm{K}}$ and $\mathrm{o}^{\prime}=\mathrm{p}\left(\mathrm{YK}, \mid \mathrm{X}_{1}, \ldots \ldots \ldots, \mathrm{X}_{\mathrm{n}}\right)$. We should choose the Sigmoid Function Eqn. -1, as a transfer function so that the ANN can handle non-linearly separately data set.

$$
0=\sigma(W . X)=\sigma\left(y_{k}\right)=\frac{1}{1+e^{-2 \times \beta \times y_{k}}}, \beta>0
$$

Where $\mathrm{X}$ is the input to the Network, $\mathrm{O}$ is the output of the network, W is the matrix of weights, and $\sigma$ is the Sigmoid Function. We implement the back propagation algorithm for the training of the weights. The back propagation algorithm is used to minimize the squared errors between the output value and the desired value as Eqn.-2. where, the outputs is the set of output units in the network, $\mathrm{D}$ is the training set, and $\mathrm{t}_{\mathrm{ik}}$ and $\mathrm{O}_{\mathrm{ik}}$ are the target and output values associated with $\mathrm{i}^{\text {th }}$ output unit and training examples $\mathrm{k}$. For a specific weight $\mathrm{w}_{\mathrm{ji}}$ in the network it is updated for each training example as follows:

$$
\begin{aligned}
& E(w)=\frac{1}{2} \sum_{k \in D} \sum_{i \in o u t p u t s}\left(t_{i k}-o_{i k}\right)^{2} \\
& \Delta w_{j i}=-\eta \frac{\partial E_{d}}{\partial w_{j i}} \\
& w_{j i}=w_{j i}+\Delta w_{j i}
\end{aligned}
$$


$\Delta w_{j i}(n)=-\eta \frac{\partial E_{d}}{\partial w_{j i}}+\alpha \Delta w_{j i}(n-1)$

where, $\eta$ is the learning rate and $\mathrm{w}_{\mathrm{ji}}$ is the weight associated with $i^{\text {th }}$ input to the network unit $\mathrm{j}$. the weight update unit $\Delta \mathrm{w}_{\mathrm{ji}}(\mathrm{n})$ depends on the $\Delta \mathrm{w}_{\mathrm{ji}}(\mathrm{n}-1)$. The new update direction of $\Delta \mathrm{w}_{\mathrm{ji}}(\mathrm{n}-1)$ is represented in Eqn. -5 .

Where $n=$ number of iterations and $\alpha=$ common momentum constant.

\section{SYSTEM DEVELOPMENT METHODOLOGIES}

The system development methodologies start with Image Acquisition and ends at Image Detection. The successfulness of this comes through the application or use of sets of image for processing, feature extraction and recognition techniques.

\section{A. Face Image Acquisition}

The process of getting image from any source, especially hardware is called as image acquisition. In the image processing because without image receiving/acquisition, the processing on the image is not possible. It is the first step in the work-flow. The sweetest Acquisition process is a digital camera in simple. Here to collect the face images, a scanner has been used. After scanning, the image can be saved into various formats such as Bitmap, JPEG, GIF and TIFF etc. This FRS can process face images of any format. The face images in the fig. 4 have been taken as sample.
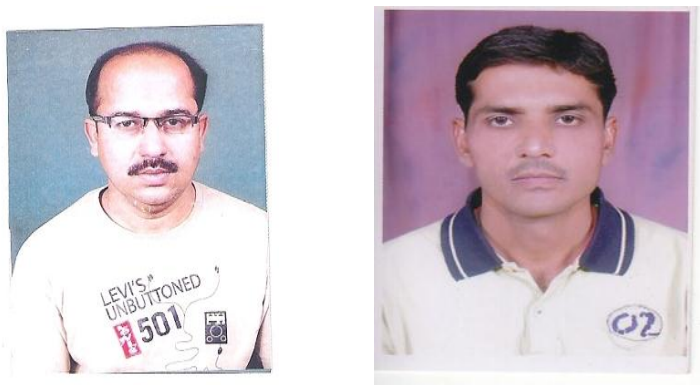

Figure-4: Sample Face Images

\section{B. Smoothing/Filtering and Clipping}

The purpose of smoothing is to reduce noise and improve the visual quality of the image often; smoothing is referred to as filtering or Smoothing. For this purpose the Smoothing filters: Gaussian Filtering Techniques is used. The weights are samples of the Gaussian function,

$$
G_{\sigma}(x, y)=\frac{1}{2 \pi \sigma^{2}} \exp ^{-\frac{x^{2}+y^{2}}{2 \sigma^{2}}}
$$

As $\sigma$ increases, more samples must be obtained to represent the Gaussian function accurately. Therefore, $\sigma$ controls the amount of smoothing. After filtering, the image is clipped to obtain the necessary data that is required for removing the unnecessary background that surrounded the image. This is done by detecting the window co-ordinates (Xmin, Ymin) and (Xmax, Ymax).


Figure-5: Face Images after Filtering and Clipping

\section{Edge Detection}

Edge detection is particularly used in the areas of feature detection and extraction of feature which aim at identifying points in a digital image at which the image brightness changes sharply or, more formally, has discontinuities.
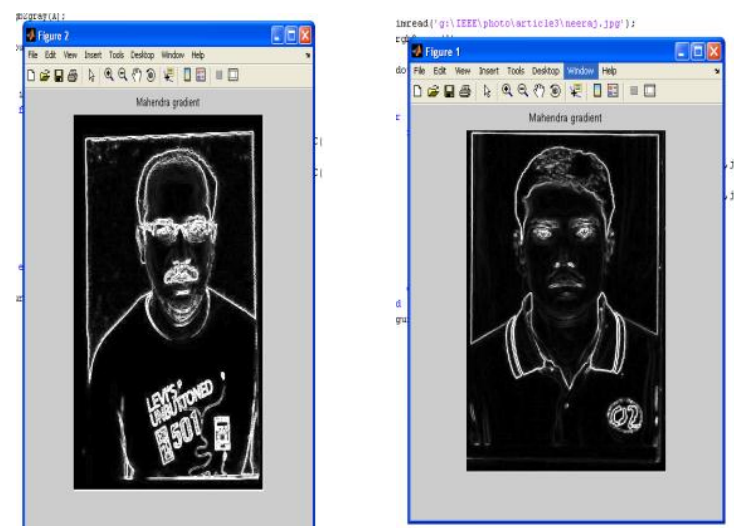

Figure-6: Edges of Face Images

\section{Image Scaling}

Image Scaling is the process of resizing of the digital image. Scaling is a non-trivial process that involves a trade-off between efficiency, smoothness and sharpness.


Figure-7: Scaling images 


\section{E. Features Extraction}

To extract features of a face at first the image is converted into a binary. From this binary image the centroid (X, Y) of the face image is calculated using equation 1 and 2 .

$$
\begin{gathered}
\mathrm{X}=\frac{\sum \mathrm{m}_{\mathrm{x}}}{\sum \mathrm{m}} \\
\mathrm{Y}=\mathrm{\sum \textrm {m } _ { \mathrm { y } }} \\
\sum \mathrm{m}
\end{gathered}
$$

Where $\mathrm{x}, \mathrm{y}$ is the co-ordinate values and $\mathrm{m}=\mathrm{f}(\mathrm{x}, \mathrm{y})=0$ or 1.Then from the centroid, only face has been cropped and converted into the gray level and the features have been collected.
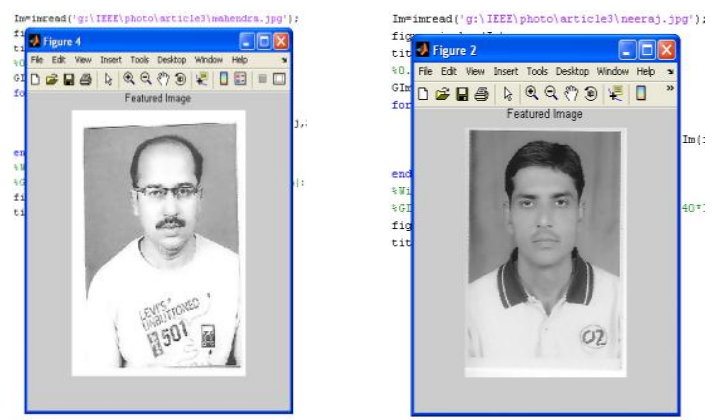

Figure-8: Features of the Faces

\section{F. Recognition}

The extracted features of the face images are fed into the GA $\&$ BPNN for recognition. Few numbers of unrecognized faces were also traced out. The result of the experiments has been interpreted through the 4.-Table-I \& 4.-Table-II.

\section{EXPERIMENTAL RESULTS}

Table I Results for GA

\begin{tabular}{|l|l|l|l|}
\hline $\begin{array}{c}\text { No. of } \\
\text { Face } \\
\text { Image }\end{array}$ & $\begin{array}{c}\text { Successfully } \\
\text { Recognized } \\
\text { Face Image }\end{array}$ & $\begin{array}{c}\text { Unrecognized } \\
\text { Face Image }\end{array}$ & $\begin{array}{c}\text { Efficiency } \\
\text { (\%) }\end{array}$ \\
\hline 5 & 3 & 2 & $60 \%$ \\
\hline 10 & 8 & 2 & $80 \%$ \\
\hline 15 & 13 & 2 & $86.67 \%$ \\
\hline 20 & 16 & 4 & $80 \%$ \\
\hline 25 & 21 & 3 & $84 \%$ \\
\hline 30 & 24 & 6 & $80 \%$ \\
\hline
\end{tabular}

Table II Results for BPNN

\begin{tabular}{|l|l|l|l|}
\hline $\begin{array}{c}\text { No. of } \\
\text { Face } \\
\text { Image }\end{array}$ & $\begin{array}{c}\text { Successfully } \\
\text { Recognized } \\
\text { Face Image }\end{array}$ & $\begin{array}{c}\text { Unrecognized } \\
\text { Face Image }\end{array}$ & $\begin{array}{c}\text { Efficiency } \\
\text { (\%) }\end{array}$ \\
\hline 5 & 3 & 2 & $60 \%$ \\
\hline 10 & 8 & 2 & $80 \%$ \\
\hline 15 & 13 & 2 & $86.67 \%$ \\
\hline 20 & 17 & 3 & $85 \%$ \\
\hline 25 & 23 & 1 & $92 \%$ \\
\hline 30 & 28 & 2 & $93.33 \%$ \\
\hline
\end{tabular}

\section{COMPARISION WITH EXISTING SYSTEMS}



Figure-9: Comparison result with GA

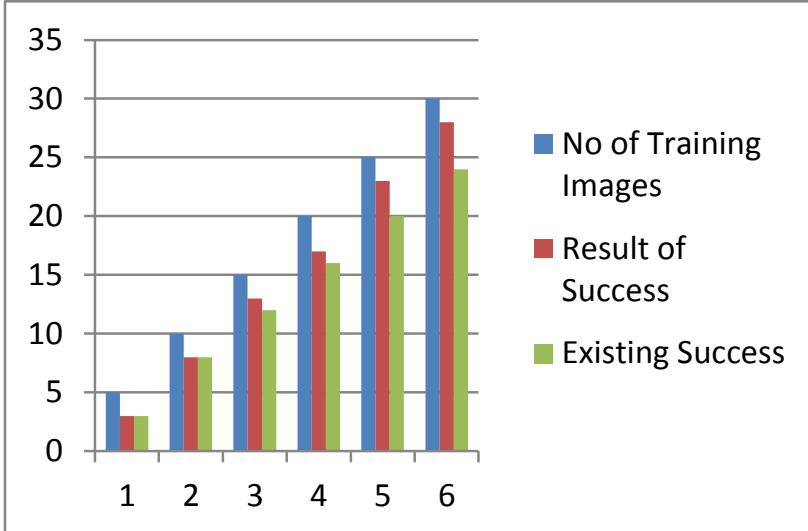

Figure-10: Comparison result with BPNN

\section{CONCLUSION}

In this paper, we have proposed a more reliable FRS approach based on the GA and the BPNN technique. Firstly, possible face regions are generated by means of the genetic algorithm and the recognition of the same was done by BPPN. This method is more robust suitable for low resolution, variable lighting and different facial expressions applied in real time video processing, single and multi threaded processing. The competence can be greater than before by using better face scanner, best technique of scaling and well-organized technique of edge detection and feature extraction of the face image. The result of the system was found to be improved one. Further better FRS can be designed if we should go with MLP (Multi-Layers Perception).

\section{REFERENCES}

[1] Introduction to Neural Networks using MATLAB 6.0, vol. 1, Tata McGraw-Hill.

[2] Rafael C. Gonzalez and Richard E Woods," Digital Image Processing Using MATLAB7”, Person Edu.

[3] S. Rajasekaran \& G.A. Vijayalakshmi Pai, "Neural Networks, Fuzzy Logic and Genetic Algorithms" PHI

[4] Jain, Fundamentals of Digital Image Processing, PHI. 
[5] S.Venkatesan and M.Karnan: Advanced Classification using Genetic Algorithm and Image Segmentation For Improved Face Detection., computer research and Development 2010 second International Conference(ICCRD)7-10 May2010 Page364-368

[6] Gur, E., Zalevsky, Z., 2007, Single-Image Digital SuperResolution A Revised Gerchberg Papoulis Algorithm, IAENG international Journal of Computer Science, 34:2, IJCS_34_2_14.

[7] Y. Suzuki, H. Saito, D. Ozawa, Extraction of the human face from natural background using GAs, Proceedings of the IEEE TENCON, Digital Signal Processing Applications, Vol. 1, 1996, pp. 221\}226.

[8] A.M. Mohamed, A. Elgammal, Face detection in complex environments from color images, Proceedings of International Conference on Image Processing 3 (1999) 622$\} 626$.

[9] Brunelli, R. and Poggio, T., "Face recognition: features versus templates," IEEE Transactions on Pattern Analysis and Machine Intelligence, Vol. 15, No. 10, pp. $1042-1052,1993$.

[10] Chellappa, R., Wilson, C.L. and Sirohey, S., "Human and machine recognition of faces: a survey," Proceedings of the IEEE, Vol. 83, No. 5, pp. 705 -741, 1995.

[11] A.Samal and P.A.Iyengar (1992): -Automatic recognition and analysis of human faces and facial expressions: A survey. Pattern Recognition.

[12] K. Okamoto, S. Ozawa, and S. Abe. A Fast Incremental Learning Algorithm of RBF Networks with Long-Term Memory. Proc. Int. Joint Conf. on Neural Networks, 102107, 2003.

[13] M.A.Turk and A.P.Petland, (1991) "Eigenfaces for Recognition," Journal of Cognitive Neuroscience. vol. 3, pp.71-86.

[14] Kailash J. Karande Sanjay N. Talbar "Independent Component Analysis of Edge Information for Face Recognition" International Journal of Image Processing Volume (3): Issue (3) pp: 120 -131

[15] Xinyu Guo, Xun Liang and Xiang Li, "A Stock Pattern Recognition Algorithm Based on Neural Networks", Third International Conference on Natural Computation, Volume 02,2007.
[16] P. M. Grant, "Artificial neural network and conventional approaches to filtering and pattern recognition", Electronics \& Communications Engineering Journal, 1989, 225.

[17] H. A. Rowley, S. Baluja, T. Kanade, "Neural NetworkBased Face Detection", IEEE Trans. On Pattern Analysis and Machine Intelligence, vol.20, No. 1, Page(s). 39-51, 1998.

[18] Fan Yang and Michel Paindavoine," Implementation of an RBF Neural Network on Embedded Systems: RealTime Face Tracking and Identity Verification", IEEE Transactions on Neural Networks, vol.14, No.5, September 2003.

[19] G.Van Dijck, M.M. Van Hulle, and M. Wevers, "Genetic Algorithm for Feature Subset Selection with Exploitation of Feature Correlations from Continuous Wavelet Transform: a real-case Application," International Journal of Computational Intelligence, 1(1) 2004,pp. 112.

[20] Kailash J. Karande Sanjay N. Talbar "Independent Component Analysis of Edge Information for Face Recognition" International Journal of Image Processing Volume (3) : Issue (3) pp: 120 -131.

\section{AUTHORS PROFILE}

Mahendra Pratap Panigrahy received the degree Master of Technology in Electronics Engineering in 2005. He is a research student of Utkal University, Orissa. Currently, he is an Associate Professor at Institute of Technology Roorkee, Roorkee. His interests are in Image Processing \& Embedded System.

Neeraj Kumar received the degree Master of Technology in Computer Science \& Engineering in 2000. He is an Associate Professor at Institute of Technology Roorkee, Roorkee. His interests are in Image Processing \& Data Structure. 